\title{
Direct visualisation of lipid bilayer cubic phases using Atomic Force Microscopy
}

Article

Accepted Version

Rittman, M., Frischherz, M., Burgmann, F., Hartley, P. G. and Squires, A. (2010) Direct visualisation of lipid bilayer cubic phases using Atomic Force Microscopy. Soft Matter, 6 (17). pp. 4058-4061. ISSN 1744-683X doi:

https://doi.org/10.1039/c002968h Available at https://centaur.reading.ac.uk/16745/

It is advisable to refer to the publisher's version if you intend to cite from the work. See Guidance on citing.

Published version at: http://dx.doi.org/10.1039/c002968h

To link to this article DOI: http://dx.doi.org/10.1039/c002968h

Publisher: Royal Society of Chemistry

Publisher statement: Reproduced by permission of The Royal Society of Chemistry (RSC)

All outputs in CentAUR are protected by Intellectual Property Rights law, including copyright law. Copyright and IPR is retained by the creators or other copyright holders. Terms and conditions for use of this material are defined in the End User Agreement.

\section{www.reading.ac.uk/centaur}

\section{CentAUR}


Central Archive at the University of Reading

Reading's research outputs online 


\title{
Direct visualisation of lipid bilayer cubic phases using Atomic Force Microscopy
}

\author{
Martyn Rittman $^{\mathrm{a}}$, Martina Frischherz ${ }^{\mathrm{b}, \mathrm{c}}$, Flame Burgmann ${ }^{\mathrm{b}}$, Patrick G. Hartley ${ }^{\mathrm{b}}$, Adam Squires ${ }^{{ }^{*}}$ \\ Received (in $X X X, X X X)$ Xth $X X X X X X X X X 200 X$, Accepted Xth $X X X X X X X X X 200 X$ \\ ${ }_{5}$ First published on the web Xth $X X X X X X X X X 200 X$ \\ DOI: 10.1039/b000000x
}

Inverse bicontinuous cubic $\left(Q_{\text {II }}\right)$ phases are nanostructured materials formed by lipid self-assembly. We have successfully imaged thin films of hydrated $\left.Q_{\text {II }}\right)$ phases from two different systems using AFM. The images show periodic arrays of water channels with spacing and symmetry consistent with published SAXS data on the bulk materials.
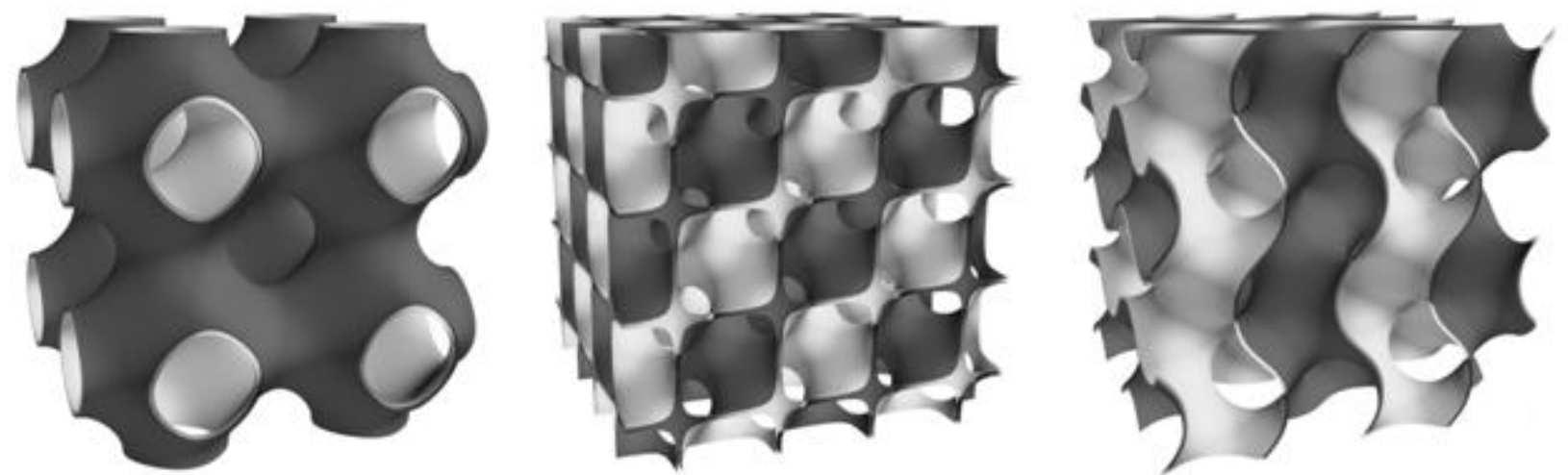

Fig. 1. Cubic phase structures. Left to right, the primitive (P), double diamond (D) and gyroid (G) triply periodic minimal surfaces. All three consist of two connected water channel networks (dark grey and white).

A number of amphiphilic systems are known to be capable of forming $\mathrm{Q}_{\text {II }}$ phases, where the amphiphile molecules form an 15 intricately curved fluid bilayer, on either side of which lie two interpenetrating continuous networks of water channels. In each $Q_{\text {II }}$ phase, a mathematical surface known as a triply periodic minimal surface lies at the middle of the lipid bilayer. Lipid systems form three $\mathrm{Q}_{\text {II }}$ phases, based on the 20 primitive $(\mathrm{P})$, double diamond (D), and gyroid (G) surfaces, and known respectively as the $\mathrm{Q}_{\mathrm{II}}{ }^{\mathrm{P}}, \mathrm{Q}_{\mathrm{II}}{ }^{\mathrm{D}}$ and $\mathrm{Q}_{\mathrm{II}}^{\mathrm{G}}$ phases (see Fig. 1). As materials, $Q_{I I}$ phases are optically transparent, stiff, visco-elastic solids ${ }^{1,2}$ that are stable in excess water.

The structures of $\mathrm{Q}_{\mathrm{II}}$ phases possess a number of 25 remarkable features that have significant potential for nanotechnological applications. They contain water channels of the order of $2-5 \mathrm{~nm}$ in diameter, whose size is extremely uniform throughout the sample, the dimensions of which may be tuned by controlling the temperature (in excess water) or 30 the lipid/water ratio (below excess water). ${ }^{3}$ The structure also provides an extremely efficient way of packing a very large contiguous surface area into a small volume. Such properties make these, and similar structurally analogous materials, promising candidates in applications where they may be 35 templated for size-selective molecular sieves or catalysts, ${ }^{4}$ or for electronic applications. ${ }^{5}$ Since the constituent lipids themselves are non-toxic, naturally-occuring biological molecules they are suitable for applications in food, 6 pharmaceuticals ${ }^{7}$ and cosmetics, ${ }^{8}$ where the bicontinuous 40 structure may be used to store and deliver both hydrophobic and hydrophilic molecules. Finally, since the bilayer is very similar to a biological cell membrane, QII phases are of biological interest, leading to their use in methodologies for membrane protein crystallization. ${ }^{9}$ Furthermore, in nature 45 these are naturally-occurring structures that perform a number of important biological roles ${ }^{10}$ and the mechanism of their formation from the lamellar phase is considered to be a model for the mechanism of bio-membrane fusion and fission. ${ }^{2}$

Much of our current understanding of $\mathrm{Q}_{\mathrm{II}}$ phases comes 50 from data obtained with small-angle $\mathrm{x}$-ray scattering (SAXS). These give the symmetry from which the different $Q_{\text {II }}$ phases may be discriminated and a very precise measurement of the lattice parameter. On the basis of SAXS experiments, we now have a good understanding of the thermodynamic and 55 geometric factors on which these depend. More recent, timeresolved synchrotron SAXS experiments ${ }^{11}$ have yielded data investigating the kinetics and mechanisms of the processes that interconvert the $\mathrm{Q}_{\mathrm{II}}$ phases and by which they are formed from the fluid lamellar phase, with direct relevance to 60 biological processes such as membrane fusion and fission. 

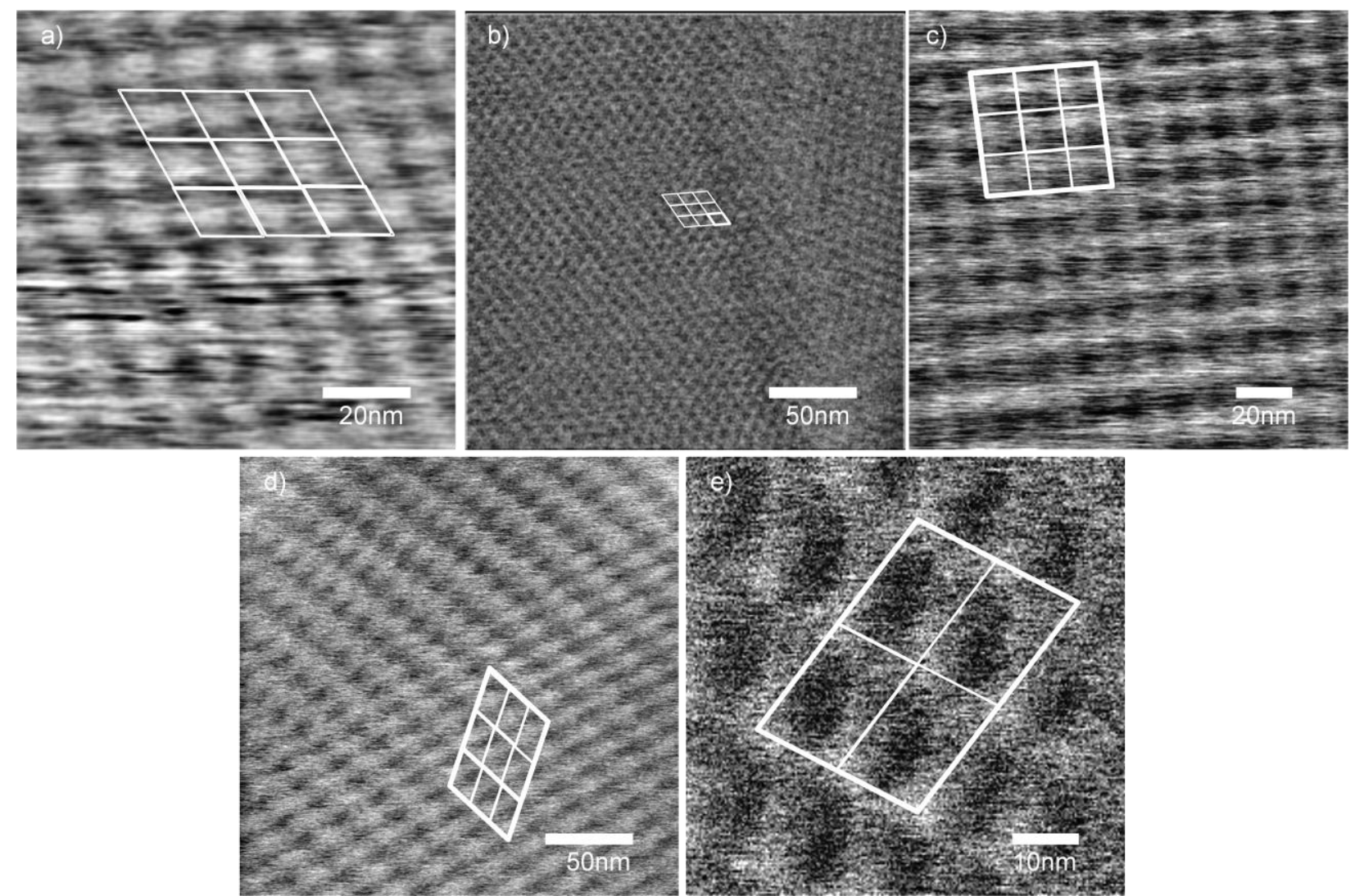

Fig 2. AFM non-contact mode images of $\mathrm{Q}_{\text {II }}$ phases in excess water. All are height images, except b) and e) which show phase. a) and d) are from monoolein and b), c) and e) from phytantriol. Unit cell lattices have been drawn on. Using the right-hand axis convention, with lattice vectors listed anticlockwise about the obtuse angle, the dimensions of these lattices are as follows: (a): $14.4 \mathrm{~nm} ; 12.8 \mathrm{~nm} ; 120^{\circ}$.(b): $8.3 \mathrm{~nm} ; 6.9 \mathrm{~nm} ; 120^{\circ}$. (c): $14.2 \mathrm{~nm} ; 14.2 \mathrm{~nm}$; $92^{\circ}$. (d): $25.0 \mathrm{~nm} ; 15.3 \mathrm{~nm} ; 115^{\circ}$. (e): $19.7 \mathrm{~nm} ; 13.6 \mathrm{~nm} ; 100^{\circ}$.

However, SAXS typically averages over many randomly oriented micron-sized domains. The data therefore provide no information on the interface of the $\mathrm{Q}_{\text {II }}$ phase with water, or of the boundary between adjacent domains; domain boundaries 10 and defects are likely to affect technological applications, visco-elastic properties ${ }^{12}$ and phase transition kinetics. ${ }^{11}$ Nor do SAXS experiments give information on the orientation of individual domains that can provide data on the epitaxy of transformations, to support or disprove various proposed 15 geometrical transformation mechanisms. ${ }^{11,13}$

A number of groups have successfully imaged individual "cubosomes", representing discrete single domains of $\mathrm{Q}_{\mathrm{II}}$ phase, using cryo-TEM. ${ }^{14}$ These particles themselves have a number of applications in, for example, drug delivery. 20 However, it is not clear to what extent they are representative of domains within a bulk polydomain sample, in particular as their stabilization requires a co-surfactant that forms an additional bilayer around each cubosome. Finally, the technique of cryo-TEM itself is a static one, and cannot be 25 used to obtain dynamic information on phase transformations.

Atomic Force Microscopy (AFM) has the potential to overcome the limitations associated with both techniques. It gives direct visualization, and has sufficient spatial resolution to image individual water channels within a single domain, 30 thus providing information on domain orientation and epitaxy, and domain boundaries (unlike bulk SAXS). It can be carried out under ambient conditions, in a temperature range consistent with equilibrium lyotropic phase formation, under water or in air, and thus has potential for structural analysis of ${ }_{35} \mathrm{Q}_{\text {II }}$ phases, both at equilibrium and during phase transitions.

Although AFM has been used extensively for single bilayers, very little work has been carried out on non-lamellar structures. ${ }^{15}$

In this work, we investigate two different systems; 140 monoolein $\left.\mathrm{CH}_{3}\left(\mathrm{CH}_{2}\right)_{7} \mathrm{CH}=\mathrm{CH}\left(\mathrm{CH}_{2}\right)_{7} \mathrm{COOCH}_{2} \mathrm{CHOHCH}_{2} \mathrm{OH}\right)$ and phytantriol $\left(\mathrm{CH}_{3}\left(\mathrm{CHCH}_{3}\left(\mathrm{CH}_{2}\right)_{3}\right)_{3} \mathrm{CCH}_{3} \mathrm{OHCHOHCH}{ }_{2} \mathrm{OH}\right)$. Both of these have well characterized phase behaviour and both form $\mathrm{Q}_{\mathrm{II}}{ }^{\mathrm{D}}$ phases at room temperature in excess water ${ }^{18}$ ${ }_{45}{ }^{19}$. We demonstrate that we can use AFM to image both of these with sufficient resolution to resolve individual water channels and that the spacing and symmetry of these are consistent with published data obtained using SAXS on bulk polydomain samples from each system.

50 Fig 2 shows AFM images of phytantriol and monoolein. We should note that due to tip broadening, the images do not give meaningful height data on individual water channels (indeed 2(b) and 2(e) are phase images). However, they do show the spacing and arrangement of the water channel 55 arrays. We have drawn unit cells onto the images, and included lattice dimensions and angles in the figure legend. Fig 2 (a) and the left hand side of (b) show features that 
appear to lie in slightly distorted hexagonal arrays, with apparent spacings of approximately $14 \pm 1 \mathrm{~nm}$ and $8 \pm 1 \mathrm{~nm}$ respectively. Fig 2 (c)-(e) show arrays with other symmetries.

Fig 2 (d) appears to show a disclination, and Fig 2 (b) 5 shows different lattices on the left and right sides of the image. Both appear to be consistent with domain boundaries, confirming our expectation that, in contrast to SAXS, AFM can be used for imaging and characterising domain boundaries of $\mathrm{Q}_{\mathrm{II}}$ phases.

10
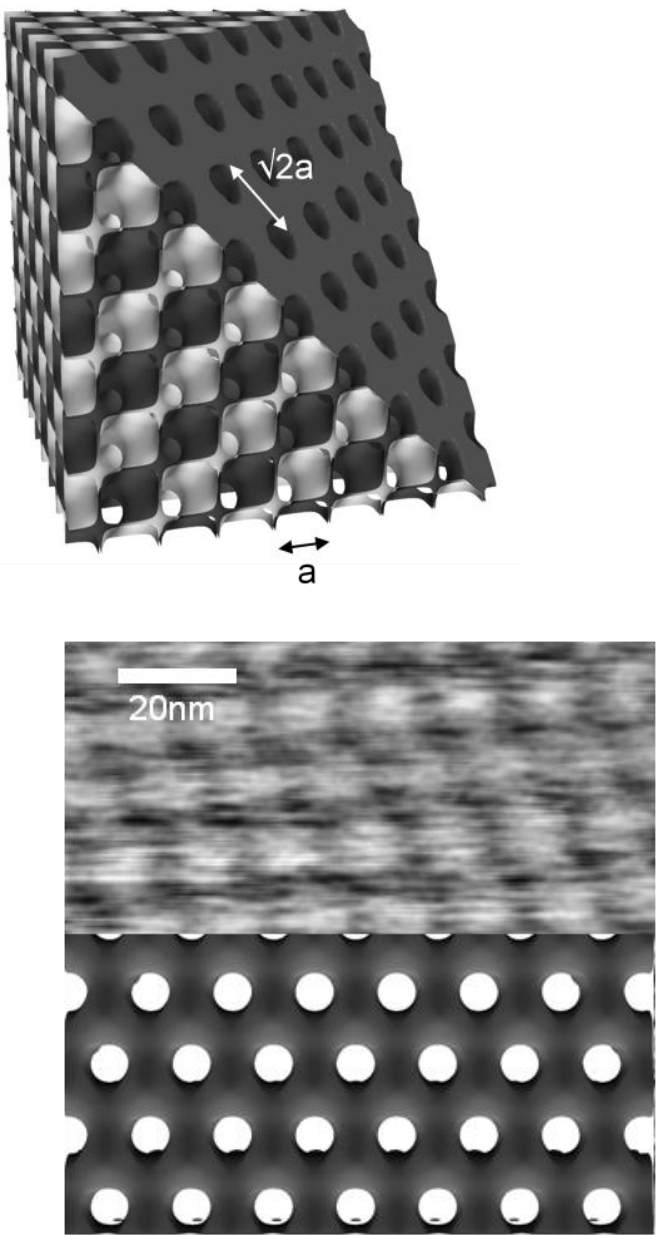

Fig.3 The (111) planes through the diamond surfaces. The light and dark surfaces represent the two water channel networks. The white channel network is capped at the surface, and the dark open to the outside, as 15 discussed in the text. Also shown are the spacings of the water channels at the surface and the lattice parameter, $a$.

The hexagonal structures shown in Fig 2 (a) and (b) are consistent with the 111 plane through the unit cell being uppermost (see Fig 3). Larsson and co-workers ${ }^{17}$ suggest that, 20 in order that the bilayer should remain continuous, one of the networks of water channels is "capped" by the bilayer, and that only the other network is open to the bulk water; this is what we expect to observe from the top surface (Fig 3). Given this assumption, we expect the spacing of features on this 25 plane to be equal to $a$ where $a$ is the lattice parameter. (Figure 3 shows a graphical justification of this; essentially, for a $\mathrm{Q}_{\mathrm{II}}$ lattice with cubic unit cells of side $a$, the spacing between features on the (111) AFM image is the distance between adjacent unit cell centres for the same colour 30 network; this is equal to the face diagonal of the unit cell, a ).

Fig. 4 Simulations of a diamond isosurface capped off at a 111 plane (lower half) and corresponding AFM images from Figures $2 \mathrm{a}$ and b (upper half). 35 Each isosurface is plotted on the same scale as its corresponding AFM image, with lattice parameter $10.0 \mathrm{~nm}$ (left) and $6.5 \mathrm{~nm}$ (right) corresponding to the literature values for lattice parameters at room temperature in excess water of monoolein and phytantriol respectively.

We can therefore estimate values for the lattice parameter $a$ for monoolein and phytantriol from Fig 2 (a) and (b), to be the feature spacing divided by . This gives values for 40 monoolein and phytantriol of $a=10 \pm 1 \mathrm{~nm}$ and $6 \pm 1 \mathrm{~nm}$ respectively. These are in good agreement with published bulk measurements of the surfactants in excess water obtained using SAXS, which are $10 \mathrm{~nm}$ for monoolein ${ }^{18}$ and 6.4-6.6 nm for phytantriol. ${ }^{19}$ There are a number of sources of error for 45 the values we have measured from AFM, including local variation of spacings, drift during AFM measurement and batch-to-batch variation of the surfactants (as compared with that used for the bulk measurements quoted).

Fig 4 shows computer simulated images corresponding to
50 the size of the AFM images in Fig 2 (a) and (b), generated with literature values of lattice parameter. These provide a visual confirmation that the hexagonal spacings observed in Fig 2 are consistent with the 111 plane.

\section{Experimental Section}

55 Phytantriol was obtained from DSM nutritional products, Germany. Glycerol mono-oleate (MO) was obtained from Sigma Aldrich, St Louis. Purities were quoted at $95 \%$ and 99\% respectively and they were used as received. Each was dissolved in chloroform (Merck, Whitehouse Station, NJ) at a ${ }_{60}$ final concentration of $10 \mathrm{mg} / \mathrm{ml}$. 
Highly ordered pyrolitic graphite (HOPG, Agar Scientific Ltd, Stansted, UK) with mosaic spread $3.5 \pm 1.5$, and size $10 \mathrm{~mm}$ by $10 \mathrm{~mm}$ was cleaved and used immediately.

Both surfactants were spin coated onto HOPG. Spin coating 5 was carried out at $2000 \mathrm{rpm}$ on a Headway Research (Garland, Texas) instrument. A single drop, approximately $10 \mu 1$, was placed into the centre of the spinning substrate from a glass pipette. The substrate was left spinning for approximately a further $30 \mathrm{~s}$ to allow for the solvent to evaporate. Successful 10 deposition was characterised by a blue/purple film visible across the substrate surface, suggesting thicknesses on the order of the wavelength of light. AFM images obtained across a gap in the coating in one film indicated a varying thickness of at least $60 \mathrm{~nm}$. Spin coated substrates were immediately 15 transferred into MilliQ water for a period of at least one hour, and overnight if possible, to hydrate.

A Digital Instruments/Veeco Multimode Nanoscope III AFM (Digital Instruments, Santa Barbara, California) was used to record height and phase tapping mode images with a $2010 \mu \mathrm{m}$ scanner unit. DNP-S10 (Veeco, Camarillo, California) AFM tips were used within the standard tapping mode fluid flow cell, in an excess of MilliQ water. Images were $512 \mathrm{x}$ 512 pixels. The nominal tip radius quoted by the manufacturer was $10 \mathrm{~nm}$.

25 AFM data were processed to highlight image features using the software provided with the instrument. Typically the images were flattened and a plane fit applied. A combination of lowpass filtering (to remove high frequency image artefacts) and contrast enhancement were also employed.

30 To generate a simulated version of surfaces of the unit cell of the diamond $\mathrm{Q}_{\text {II }}$ surface, the following equation was used: ${ }^{16}$

where are the coordinates of a point in the unit cell and is a parameter controlling the volume of the resultant structure. An isosurface ( ) is the $\mathrm{Q}_{\mathrm{II}}$ structure 35 where the top surface is the ' ' section.

Coordinates for isosurfaces through the unit cell of a diamond lattice were calculated using Python 2.5 (including Scipy 0.6) and plotted using Mayavi2.

\section{Conclusions}

40 In conclusion, we have demonstrated that we can produce thin films of $\mathrm{Q}_{\mathrm{II}}$ phase, and use these to image the water channels within using AFM. The data show good agreement with published behaviour of bulk $\mathrm{Q}_{\mathrm{II}}$ phase samples measured by SAXS, showing the orientation of single domains, which 45 could not be done with bulk SAXS measurements, and supporting a proposed model for the interface with water. AFM on thin $\mathrm{Q}_{\mathrm{II}}$ films clearly shows potential for resolving a number of outstanding questions relating to domain size, structure and epitaxy in such systems.

\section{Acknowledgements}

We thank Prof. Marcus Textor, ETH Zurich, for facilitating Martina Frischherz's visit to CSIRO, Australia, and Celesta ${ }_{55}$ Fong at CSIRO for helpful discussions.

\section{Notes and references}

${ }^{a}$ Department of Chemistry, University of Reading, Whiteknights, Reading, RG6 6AH, UK; E-mail: a.m.squires@ reading.ac.uk ${ }^{b}$ CSIRO Molecular \& Health Technologies, Bag 10, Clayton South, 60 Victoria 3169, Australia.

${ }^{c}$ BioInterface Group, Laboratory for Surface Science and Technology, ETH Zurich, CH-8093 Zurich, Switzerland

1 P. Spicer, K. Hayden, M. Lynch, A. Ofori-Boateng, and J. Burns, Langmuir, 2001, 17, 5748-5756.

652 D. P. Siegel and M. Kozlov, Biophys J, 2004, 87, 366-374.

3 R. H. Templer, J. M. Seddon, N. A. Warrender, A. Syrykh, Z. Huang, R. Winter, and J. Erbes, J Phys Chem B, 1998, 102, 7251-7261.

4 R. Holyst, Nat Mater, 2005, 4, 510-1.

5 V. N. Urade, T. Wei, M. P. Tate, J. D. Kowalski, and H. W. Hillhouse, 70 Chem Mater, 2007, 19, 768-777.

6 R. Mezzenga, P. Schurtenberger, A. Burbidge, and M. Michel, Nat Mater, 2005, 4, 729-40.

7 C. J. Drummond and C. Fong, Curr Opin Colloid In, 1999, 4, 449-456.

8 P. Spicer, M. Lynch, S. Hoath, and M. Visscher, in Delivery system 75 handbook for personal care and cosmetic products: technology, applications, and formulations Breakthroughs in personal care and cosmetic technology, M. R. Rosen, William Andrew, 2005, pp. 604-620.

9 M. L. Chiu, P. Nollert, M. Loewen, H. Belrhali, E. Pebay-Peyroula, J. Rosenbusch, and E. Landau, Acta Crystallogr D Biol Crystallogr, 2000, 80 56, 781-784.

10 V. Luzzati, Curr Opin Struc Biol, 1997, 7, 661-668.

11 A. Squires, R. H. Templer, J. M. Seddon, J. Woenkhaus, R. Winter, T. Narayanan, and S. Finet, Phys Rev E, 2005, 72, 11502.

12 J. Jones and T. McLeish, Langmuir, 1995, 11, 785-792.

8513 D. P. Siegel, Biophys J, 2008, 95, 5200-15.

14 J. Gustafsson, H. Ljusberg-Wahren, M. Almgren, and K. Larsson, Langmuir, 1996, 12, 4611-4613.

15 C. Neto, G. Aloisi, P. Baglioni, and K. Larsson, Journal of Physical Chemistry B, 1999, 103, 3896-3899.

9016 S. Andersson, M. Jacob, S. Lidin, and K. Larsson, Z Kristallog, 1995, $210,315-318$

17 K. Larsson, Curr Opin Colloid In, 2000, 5, 64-69. 
18 J. Briggs, H. Chung, and M. Caffrey, J. Phys. II France, 1996, 6, $723-$ 751.

19 J. Barauskas and T. Landh, Langmuir, 2003, 19, 9562-9565.
20 A. Squires, R. H. Templer, J. M. Seddon, J. Woenckhaus, R. Winter, 5 S. Finet, and N. Theyencheri, Langmuir, 2002, 18, 7384-7392. 